\title{
Retinal vein occlusion following intravenous immunoglobulin treatment
}

\author{
B M T P Nawasiwatte 1 , S C Somaratne ${ }^{2}$, A Fernando², P S Gunaratne ${ }^{2}$
}

Ceylon Medical Journal 2012; 57: 170-171

\section{Introduction}

Intravenous immunoglobulin (IVIg) is concentrated human immunoglobulins made from pooled donor plasma. Commercial preparations were used for clinical administration since early 1980s [1]. It modulates the immune response in a variety of inflammatory and autoimmune disorders. Although high-dose IVIg generally is considered safe, it is not without side effects and may promote life-threatening thrombosis [2]. We describe a young patient with Guillain Barré syndrome (GBS) who experienced retinal vein occlusion (RVO) following IVIg therapy.

\section{Case report}

A 27-year old, previously healthy, unmarried Sri Lankan female presented with progressive, ascending symmetrical weakness of lower limbs for two days which was preceded by a lower respiratory tract infection. She had flaccid, areflexic limbs with grade 2-3 power both proximally and distally but remained haemodynamically stable with intact sphincters and all modalities of sensation.

Over the next two days, weakness progressed to involve the upper limbs, bulbar muscles and bilateral facial nerves but external ocular muscles were spared. Her fundoscopy was normal. All basic haematological and biochemical tests (ESR, CRP, renal and liver profile, FBC) were normal. Clinical diagnosis of GBS was supported by the demyelinating polyneuropathy evident on nerve conduction test. Intra venous immunoglobulin was commenced according to the standard protocol $(0.4 \mathrm{~g} / \mathrm{kg} /$ day). She had never received IVIg before and did not develop any acute reactions. A total dose of $100 \mathrm{~g}$ IVIg was given during next 5 days following which she started to improve clinically never requiring artificial ventilation. She was given prophylactic anticoagulation to prevent deep vein thrombosis secondary to immobilisation. Lumbar puncture done on the tenth day showed cell protein dissociation with normal opening pressure. She commenced physiotherapy as an in-ward patient.

While the paralysis was improving, on day 18 (14 days after starting IVIg) she complained of diplopia and fading vision. On examination her visual acuity (VA) was
$6 / 12,6 / 12$ with normal visual fields. There was no ophthalmoplegia but both fundi showed marked disc oedema with haemorrhages. A clinical diagnosis of RVO was suspected and a prompt ophthalmological referral was arranged. It was reported as a variant of central retinal vein occlusion with dilated veins due to hyperviscosity.

All basic investigations were repeated and ESR alone was grossly elevated to $115 \mathrm{~mm} / 1$ st hour with normal CRP, serum proteins and albumin. The thrombophilic screening (coagulation profile, ANA, anti lupus antibody), thyroid function tests, serum lipid and sugar levels were normal. HIV and VDRL screenings were negative and chest X ray, ultrasound abdomen, carotid and renal Doppler studies and 2D echo cardiography also were normal. Blood picture showed moderate rouleaux formation. There was no evidence of infection. The MRI and the MRV of the brain were normal.

She was managed supportively with regular VA assessments. As both VA and limb weakness were improving she was sent home on day 25 . On day 32 she walked unsupported and VA has improved to 6/9 but ESR was $110 \mathrm{~mm} /$ hour. The repeat CSF analysis on day 42 was normal and fundal oedema and haemorrhages have improved. The ESR has come down to $30 \mathrm{~mm} /$ hour. Patient is currently being followed up as an outpatient.

\section{Discussion}

Though generally considered safe, IVIg treatment can cause thrombotic complications [1]. Five cases of stroke, two cases of deep vein thrombosis, seven myocardial infarctions, one case each of RVO and pulmonary embolism have so far been described with IVIg [1-7]. The rate of thrombotic complications following IVIg may be as high as 3-5\% though underreported and can involve both arterial and venous circulation [8]. In this case, though GBS itself can rarely cause thromboembolic phenomena, the sequence of events favour IVIg as the cause.

The mechanism of thrombosis secondary to IVIg is unknown but it is attributed to the resultant hyperviscosity of blood, as IVIg can cause remarkable biochemical and hematological changes $[2,9,10]$. Furthermore such changes are often confused with a serious medical problem such

${ }^{1}$ Department of Neurology, National Hospital of Sri Lanka, Colombo.

Correspondence: BMTPN, e-mail: <thusha.nawasiwatte@gmail.com>. Received 25 April and revised version accepted 23 June 2012. Competing interests: none declared. 


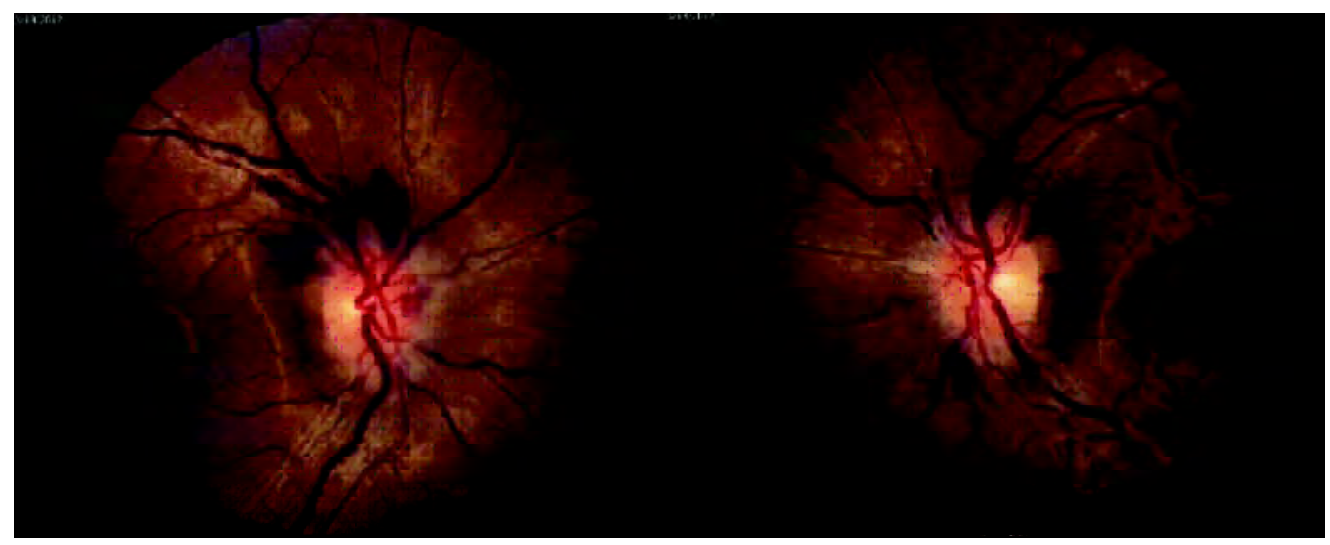

Figure 1. Fundal photography on day 1 of visual deterioration (day 14 post IVIg).

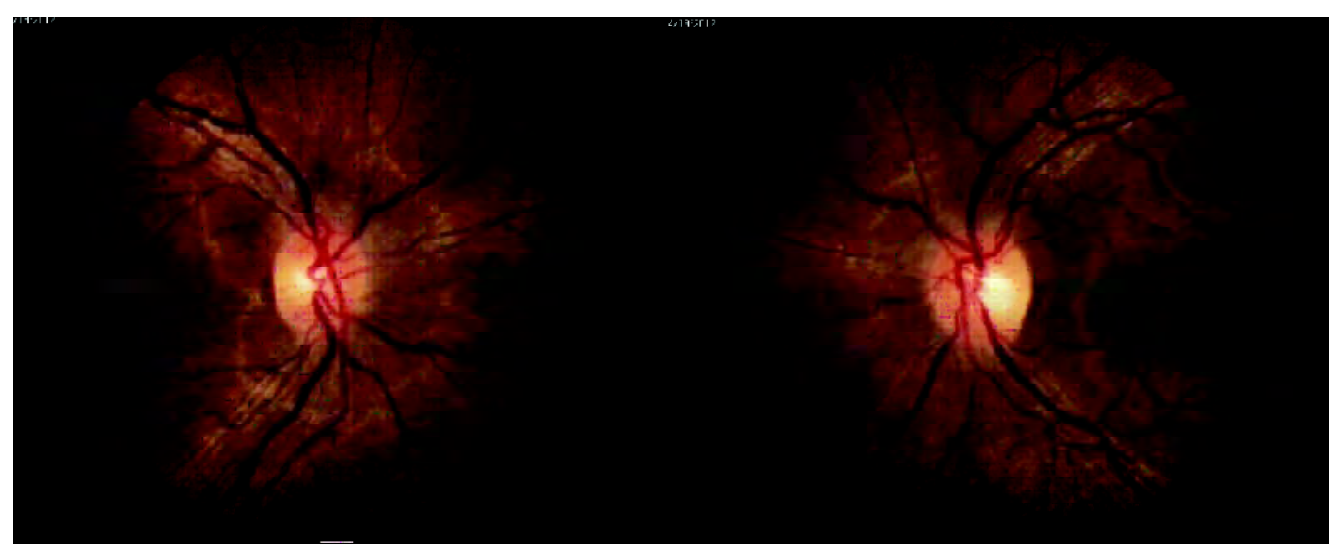

Figure 2. Day 42 post IVIg.

as disease reactivity. ESR is elevated due to enhanced rouleaux formation, polymerisation between proteins and reduced surface area caused by the infused IVIg [10]. In addition, IVIg reportedly cause platelet activation and arterial vasospasm $[2,4]$. These viscous effects are dose dependent and can last for weeks, increasing susceptibility to thromboembolism $[9,10]$. Across the world, irrespective of brands IVIg related thrombotic complications have been reported, generally occurring between 1-14 days of the therapy $[4,5]$.

\section{References}

1. Emerson GG, Herndon CN, Sreih AG. Thrombotic Complications after Intravenous Immunoglobulin Therapy in Two Patients (http://emedicine.medscape.com)

2. Woodruff RK, Grigg AP, Firkin FC, Smith IL. Fatal thrombotic events during treatment of autoimmune thrombocytopenia with intravenous immunoglobulin in elderly patients [letter]. Lancet 1986; 2: 217-8.

3. Brannagan TH, Nagle KJ, Lange DJ, Rowland LP. Complications of intravenous immune globulin treatment in neurologic disease. Neurology 1996; 47: 674-7.

4. Voltz R, Rosen FV, Yousry T, Beck J, Hohlfeld R. Reversible encephalopathy with cerebral vasospasm in a Guillain-Barré syndrome patient treated with intravenous immunoglobulin. Neurology 1996; 46: 250-1.

5. Oh KT, Boldt C, Danis RP. Iatrogenic central retinal vein occlusion and hyperviscosity associated with high-dose intravenous immunoglobulin administration. American Journal of Ophthalmology 1997; 124: 416- 8.

6. Alliot C, Rapin JP, Besson M, Bedjaoui F, Messouak D. Pulmonary embolism after intravenous immunoglobulin. Journal of Royal Society of Medicine 2001; 94: 187-8.

7. Elkayam O, Paran D, Milo R, et al. Acute myocardial infarction associated with high-dose intravenous immunoglobulin infusion for autoimmune disorders: a study of four cases. Annals of Rheumatic Diseases 2000; 59:77-80.

8. Haplea SS, Farrar JT, Gibson GA, et al. Thromboembolic events associated with intravenous immunoglobulin therapy [abstract]. Neurology 1997; 48: A54.

9. Reinhart WH, Berchtold PE. Effect of high-dose intravenous immunoglobulin therapy on blood rheology. Lancet 1992; 339: $662-4$.

10. Lee KY, Lee HS, Hong JH, et al. High dose intravenous immunglobulin downregulates the activated levels of inflammatory indices except erythrocyte sedimentation rate in acute stage of Kawasaki Disease. Journal of Tropical Pediatrics 2005; 51: 98-101. 